\title{
Organization, evolution and function of fengycin biosynthesis gene clusters in the Bacillus amyloliquefaciens group
}

Qingchao Zeng ${ }^{1,2}$, Jianbo Xie ${ }^{2}$, Yan Li ${ }^{1}$, Xinyi Chen ${ }^{1}$, Xiaofei Gu${ }^{1}$, Panlei Yang ${ }^{1}$, Guangcan $\mathrm{Hu}^{3}$ and Qi Wang ${ }^{\text {** }}$

\begin{abstract}
The Bacillus velezensis strain PG12, belonging to the Bacillus amyloliquefaciens group, is an endophytic bacterium known for its antimicrobial activities against crop pathogens. However, our knowledge of the molecular basis underlying its biocontrol activity and the relatedness of different strains in the Bacillus amyloliquefaciens group is limited. Here, we sequenced and analyzed the genome of PG12 to test its taxonomic affiliation and identified genes involved in the biocontrol activity. The phylogenomic analysis results indicate that PG12 belongs to $B$. velezensis, a subgroup of the $B$. amyloliquefaciens group. By comparing the genomes of 22 strains in this group, we confirmed that it comprises three different phylogenetic lineages: B. amyloliquefaciens, B. velezensis and B. siamensis. Three secondary metabolism gene clusters related to the production of lipopeptides, namely fengycin, iturin and surfactin, were identified in the genomes of the $B$. amyloliquefaciens group. The core genome of $B$. velezensis is enriched in secondary metabolism genes compared with $B$. siamensis and $B$. amyloliquefaciens. Three of the five genes pertaining to the gene cluster responsible for fengycin biosynthesis (fen $B C D$ ) were found in $B$. velezensis and $B$. siamensis, but not in B. amyloliquefaciens. Phenotypic analysis showed that the $\triangle$ fenA mutant of PG12 displayed significantly decreased biofilm formation and swarming motility, which indicates that fengycin contributes to the colonization and pathogen control abilities of PG12. Our results also suggest that B. siamensis and B. velezensis have acquired the fenBCD genes from Paenibacillus spp. by horizontal gene transfer ( $H G T$ ). Taken together, the results provide insights into the evolutionary pattern of the B. amyloliquefaciens group strains and will promote further researches on their taxonomy and functional genomics.
\end{abstract}

Keywords: Bacillus amyloliquefaciens, Biocontrol, Comparative genomic, Evolution, Genome sequencing

\section{Background}

Pathogenic microorganisms affecting plant health are a major and chronic threat to food production worldwide (Compant et al. 2005). The annual yield loss of crop plants due to microbial diseases is approximately $25 \%$. To reduce crop loss, farmers rely heavily on agrochemicals (Schäfer and Adams 2015; Wu et al. 2015). However, overuse of chemicals to enhance crop yield and control

\footnotetext{
*Correspondence: wangqi@cau.edu.cn

${ }^{1}$ Department of Plant Pathology, MOA Key Lab of Pest Monitoring

and Green Management, College of Plant Protection, China Agricultural University, Beijing 100193, China

Full list of author information is available at the end of the article
}

plant diseases has resulted in an irreversible loss of soil quality along with serious health and environmental problems (Prashar et al. 2013). As a result, many countries are now restricting the use of a wide range of fungicides and pesticides (Rahman 2013). In this changing context, disease control in plants by beneficial bacteria is steadily increasing as an alternative to chemical pesticides (Wu et al. 2015). Several bacterial species, such as Pseudomans spp., Bacilllus spp. and Streptomyces spp. have been commercialized as biological control agents (Paterson et al. 2017). Among them, Bacillus spp. have become increasingly important in agriculture original author(s) and the source, provide a link to the Creative Commons licence, and indicate if changes were made. The images or other third party material in this article are included in the article's Creative Commons licence, unless indicated otherwise in a credit line to the material. If material is not included in the article's Creative Commons licence and your intended use is not permitted by statutory regulation or exceeds the permitted use, you will need to obtain permission directly from the copyright holder. To view a copy of this licence, visit http://creativecommons.org/licenses/by/4.0/. 
and agro-food industry where spore-forming strains are favored due to their long-term viability (Qiao et al. 2014).

The genus Bacillus represents a large group of Grampositive bacteria belonging to the Firmicutes phylum. They are capable of forming stable dormant structures called endospores in nutrient-poor and stressful environmental conditions (Hamdache et al. 2013). The bacteria from this group can inhabit a large variety of ecological niches, including soil, water, plant surfaces and rhizosphere (Siefert et al. 2000; Feng et al. 2014; Kim et al. 2015; Gao et al. 2019). B. cereus, B. subtilis and B. amyloliquefaciens are three Bacillus species known for supporting plant growth and protecting plants from diseases (Chen et al. 2016; Fan et al. 2017b; Zeng et al. 2018). The biocontrol activity mediated by Bacillus can be divided into direct and indirect forms. Effective biological control of pathogens by Bacillus is achieved by a combination of mechanisms, including production of antibiotic, induction of host resistance and promotion of plant growth (Paterson et al. 2017; Jayapala et al. 2019). Among them, the production of antibiotics is one of the primary mechanisms for achieving biocontrol effect (Li et al. 2021). The antibiotic activity of Bacillus species is partly driven by the secretion of active lipopeptides. These cyclic lipopeptides belong to three main families: surfactin (surfactin, lychenisin, pumilacidin and halobacilin), iturin (iturin, bacillomycin and mycosubtilin) and fengycin (fengycin, plipastatin and maltacin) (Zhao et al. 2017; Fira et al. 2018). They are produced by nonribosomal peptide synthetase and exhibit broad-spectrum antimicrobial activity (Cochrane and Vederas 2016). Different lipopeptides can act synergistically to inhibit the growth of phytopathogens (Koumoutsi et al. 2014). In addition, studies have revealed that lipopeptides play an important role in maintaining colonization behaviors such as biofilm formation and swarming motility (Luo et al. 2014; Fan et al. 2017b).

According to phylogenomic analyses, the $B$. amyloliquefaciens group comprises three species: $B$. siamensis, mainly occurring in Asian food, some of which are capable of controlling phytopathogens (Fan et al. 2017a, 2019); B. velezenisis, the main source of bioformulations used in organic agriculture; B. amyloliquefaciens, known for its ability to produce industrial enzymes (Wu et al. 2015; Fan et al. 2017a). Currently, many basic characteristics of these species remain unclear. With the advent of comparative genomics and the availability of an increasing number of whole genome sequences, it is possible to understand the B. amyloliquefacies group strains at genome level. In our previous study, we isolated a B. velezensis strain PG12 from apple fruit, which exhibits distinctive inhibition to apple ring rot pathogen and broad-spectrum antagonistic activities against other pathogens. PG12 demonstrates an outstanding biocontrol performance, especially against Botryosphaeria dothidea. It produces several antagonistic compounds, including iturin and fengycin (Chen et al. 2016). Recently, the released data on genome sequences of numerous Bacillus strains have to some extent revealed the underlying molecular basis of their biocontrol performance (Kim et al. 2015; Li et al. 2021).

Based on the above background, the aims of the present study were to investigate: (1) the genomic features of PG12 and its phylogenetic relationship; (2) the significant genomic differences between the $B$. amyloliquefaciens group strains; (3) the difference in the specific genes involved in biocontrol activity between the $B$. amyloliquefaciens group strains. Consequently, we sequenced and analyzed the genome of PG12, and meanwhile, we collected previously published genomes of the B. amyloliquefaciens group strains to perform a comparative genomic analysis. The results will expand our knowledge about the $B$. amyloliquefaciens group strains, with emphasis on their taxonomical status. Understanding the genomic features enables us to gain novel insights into the ecology and evolution of the B. amyloliquefaciens group.

\section{Results}

\section{Genome features of $B$. velezensis PG12}

To identify genetic factors involved in the biocontrol activity of $B$. velezensis PG12, the genome of this strain was sequenced and analyzed. The genome assembly is $3,990,845$ bp with 22 scaffolds $\left(\mathrm{N}_{50}=2,085,242 \mathrm{bp}, \mathrm{G}+\mathrm{C}\right.$ content of $46.45 \%)$. The number of predicted proteincoding genes is 3,884 , including 3,001 genes with a putative function and 883 hypothetical protein-encoding genes (Additional file 1: Figure S1). These protein-coding genes have an average length of $917 \mathrm{bp}$ and account for $89.25 \%$ of the PG12 genome. Furthermore, a total of 56 tRNA-coding genes and 8 rRNA genes were predicted in the genome sequence (Table 1 ).

\section{Phylogenomic analysis of $B$. velezensis PG12}

Preliminary molecular identification based on $16 \mathrm{~S}$ rDNA and gyrA sequences assigned PG12 strains to $B$. amyloliquefaciens (Chen et al. 2016). Here, we performed a phylogenomic reconstruction based on 624 singlecopy conserved genes using the maximum-likelihood (ML) method. PG12 was clearly clustered within the $B$. velezensis clade, with $B$. velezensis CAU B946 as its closest relative (Fig. 1). They have an average nucleotide identity (ANI) of 99.96\% (Additional file 2: Table S1).

Genome comparison of several B. velezensis strains, including PG12, revealed a high sequence conservation throughout the genome of these strains (Additional file 1: 
Table 1 Genomic features of B. velezensis PG12

\begin{tabular}{lll}
\hline Attribute & PG12 & \\
\cline { 2 - 3 } & Value & \% of total \\
\hline Genome size (bp) & $3,995,119$ & 100 \\
DNA coding region (bp) & $3,562,014$ & 89.16 \\
Total genes & 3,956 & 100 \\
Protein-coding genes & 3,884 & 98.17 \\
Protein-coding genes with function prediction & 3,002 & 75.88 \\
Protein-coding genes assigned to COGs & 2,920 & 73.81 \\
rRNA genes & 11 & 0.28 \\
tRNA genes & 60 & 1.52 \\
\hline
\end{tabular}

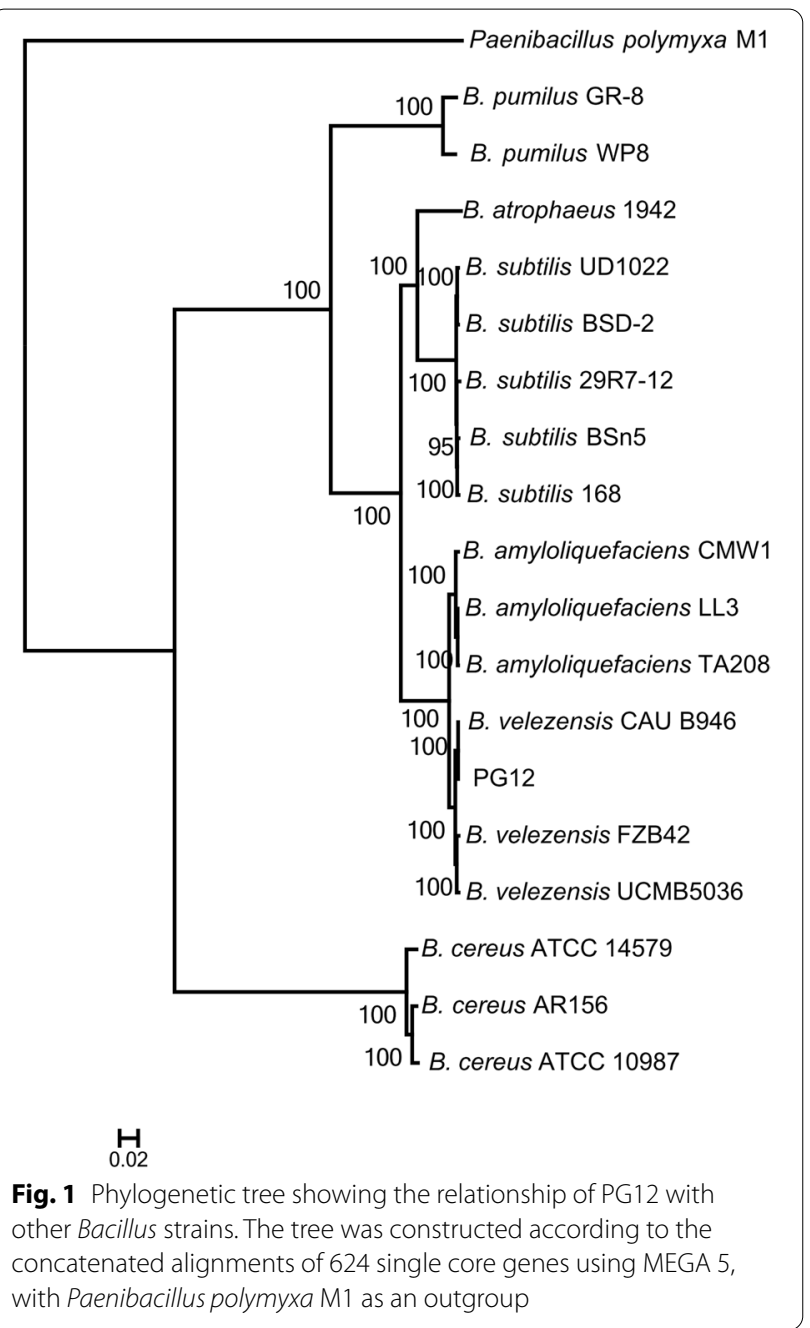

Figure S2). This was confirmed by the pan-genome analysis carried out among four B. velezensis strains. Genomewide comparisons of orthologous clusters between PG12 and closely related B. velezensis strains indicated that these B. velezensis strains contained 3,226 genes in their core genome, covering from $83.1 \%$ to $88.7 \%$ of their genomes (Additional file 1: Figure S3).

\section{Distinct phylogenetic and evolutionary history of the $B$. amyloliquefaciens group strains}

A phylogenetic tree of the B. amyloliquefaciens group strains based on 860 conserved single-copy genes was constructed, and the result showed that this group strains were clearly separated into three different phylogenetic lineages, i.e. B. amyloliquefaciens, B. velezensis and $B$. siamensis (Additional file 1: Figure S4), consistent with previous reports (Fan et al. 2017a; Chun et al. 2019). Our ANI analysis further supports the distribution of $B$. amyloliquefaciens group strains in three clusters (Fig. 2).

\section{Genes involved in secondary metabolism are enriched in $B$. velezensis strains}

The pan-genome analysis of 22 B. amyloliquefaciens group strains was conducted and yielded a pan-genome with 7,753 genes. The core, accessory and unique genomes comprise 2,806, 2,287 and 2,660 genes, respectively. The strain-specific genes for these selected $B$. amyloliquefaciens group strains ranged from 37 to 324 (Fig. 3). Meanwhile, the sizes of pan-genome and coregenome of B. velezensis, B. siamensis and B. amyloliquefaciens were estimated using the above genome data. $B$. velezensis strains have a core genome of 3,138 orthologous genes $(76.8 \%-86.3 \%$ of the protein repertoire); while B. amyloliquefaciens and B. siamensis strains have a core genome of $3,116(73.9 \%-81.6 \%$ of the protein repertoire) and 3,144 orthologous genes $(73.1 \%-85.8 \%$ of the protein repertoire), respectively. The pan-genome sizes of B. velezensis, B. siamensis and B. amyloliquefaciens appeared to reach infinity when the number of added genomes increased infinitely, suggesting that these species have open pan-genomes (Additional file 1: Figure S5). Furthermore, we compared the core genome among the $B$. amyloliquefaciens group strains by using the Cluster of Orthologous Group of proteins (COG) assignments to determine whether there are differences in the proportion of the core genome attributable to particular cellular processes. The core genomes of $B$. velezensis strains were found to be highly enriched in secondary metabolism genes compared with those of $B$. amyloliquefaciens and B. siamensis strains (Table 2) (Fisher's exact test; $P<0.05$ ).

\section{The distribution of lipopeptide gene clusters in the $B$. amyloliquefaciens group strains}

Three gene clusters coding for the cyclic lipopeptides: surfactin, bacillomycin and fengycin were identified in the genomes of the $B$. amyloliquefaciens group strains (Fig. 4). The most obvious difference between the 


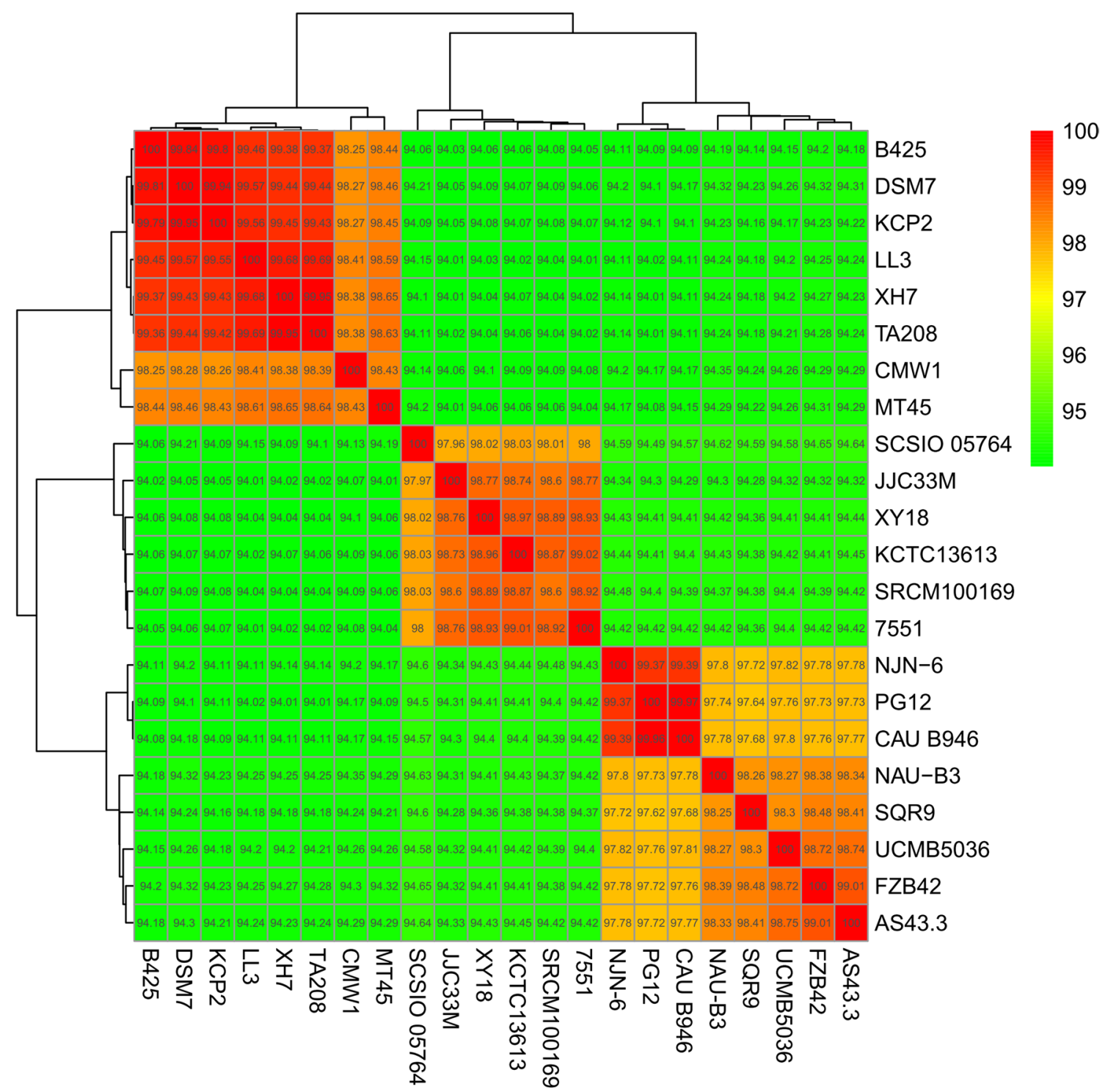

Fig. 2 Heat-map based on average nucleotide identity values calculated between genomes of 22 strains of the B. amyloliquefaciens group

aforementioned three subgroup strains was found in the gene cluster coding for fengycin. The fengycin gene cluster $(f e n A, f e n B, f e n C, f e n D$ and $f e n E)$ is conserved in all the $B$. velezensis and $B$. siamensis strains that we tested. In contrast, only two genes ( $f e n A$ and $f e n E$ ) were found in B. amyloliquefaciens strains (Fig. 4 and Additional file 1: Figure S6). By contrast, the surfactin and iturin gene clusters are highly conserved in the B. amyloliquefaciens group, and only slight differences were noticed between different subgroup strains (Fig. 4).

In all the examined $B$. velezensis and $B$. siamensis strains, we observed striking difference $(P<0.01)$ in the $\mathrm{G}+\mathrm{C}$ content between the average genome (46.36\%) and $f e n B C D$ gene sequences $(49.77 \%, 51.70 \%$ and $50.82 \%$ for $f e n B$, fenC and $f e n D$, respectively) (Additional file 1: Figure S7). The observed bias in $\mathrm{G}+\mathrm{C}$ content of $f e n B C D$ suggests a potential HGT might occur in these genes (Xie et al. 2014). To gain insights into the origin of $f e n B C D$ genes in B. velezensis strains, we constructed phylogenetic trees based on the protein sequence of fenB, fenC or fenD (Additional file 1: Figure S8-S10). The fenB, fenC and fenD sequences of B. velezensis clustered with their orthologs from Paenibacillus spp., suggesting a transfer of the fengycin gene cluster from Paenibacillus spp. to B. velezensis. 


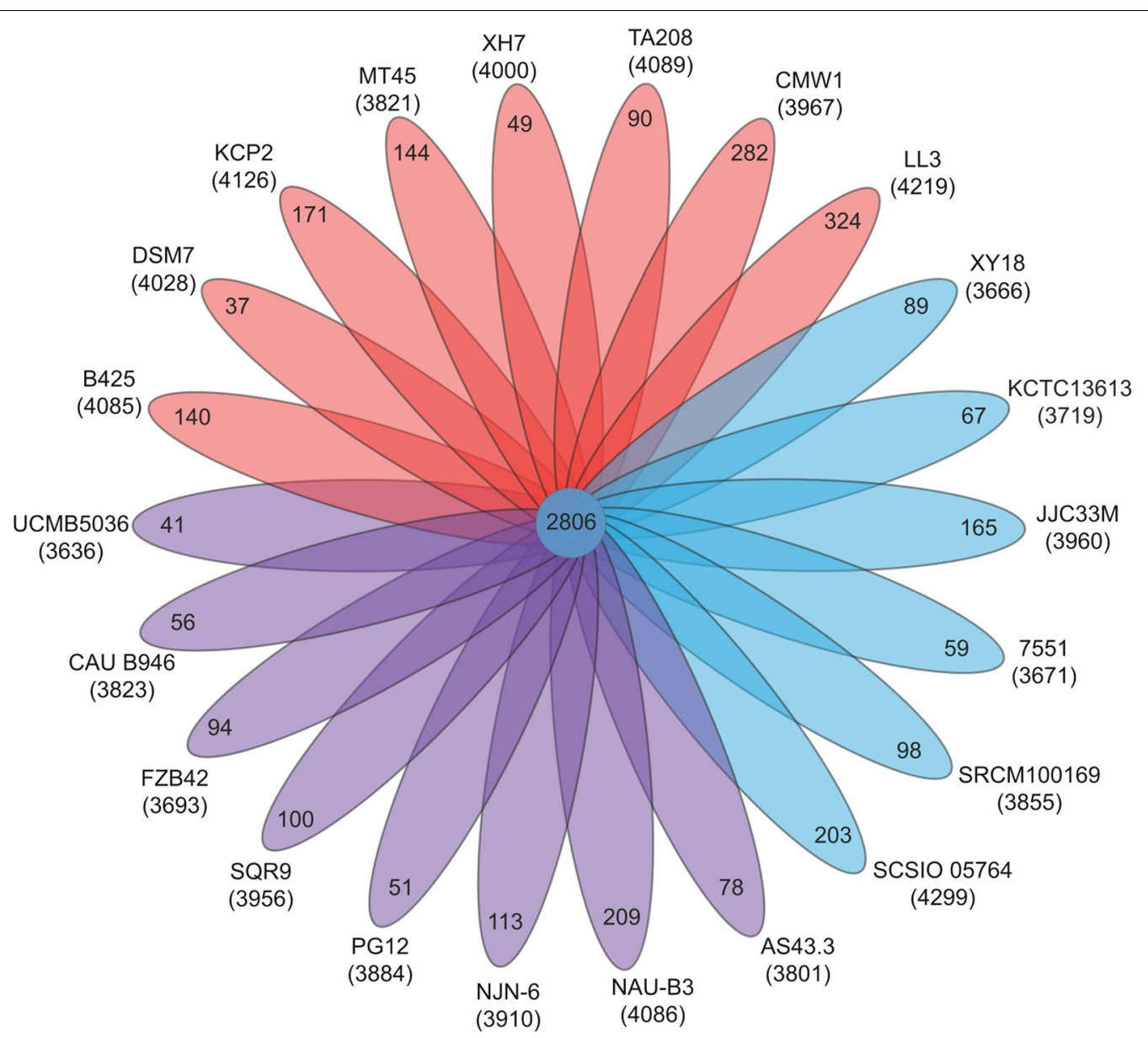

Fig. 3 Genomic diversity of the B. amyloliquefaciens group strains. Each strain is represented by a colored oval: B. velezensis (purple), $B$. amyloliquefaciens (red) and B. siamensis (blue). The number of orthologous coding sequences (CDS) shared by all strains (the core genome) is in the center. Overlapping regions show the number of CDSs conserved only within the specified genomes. Numbers in non-overlapping portions of each oval indicate the number of CDSs unique to each strain. The total number of protein-coding genes within each genome is listed below the strain name

The fenA mutant exhibited decreased swarming motility, biofilm formation and antimicrobial activities

In B. subtilis, swarming motility and biofilm formation are both considered as important phenotypic features favoring lipopeptide secretion (Allard-Massicotte et al. 2016; Fan et al. 2017b). To investigate if the biosynthesis of fengycin also affects these features, we produced a $\triangle$ fenA mutant from the parental strain PG12. Compared with the wild-type PG12 strain, the $\triangle$ fenA mutant exhibited a substantial reduction in its swarming motility (Fig. 5a, d). PG12 formed colonies with dense wrinkles and compact structure on MSgg plate, and a thick and wrinkled floating pellicle in MSgg broth. In contrast, the $\triangle$ fenA mutant formed a flat colony on MSgg plate and a thin and less wrinkled floating layer in MSgg broth (Fig. 5b, c, e). Thus, deletion of the fenA gene significantly influenced the biofilm formation and motility of PG12. In addition, the $\triangle f e n A$ mutant showed a decreased ability to inhibit the growth of plant pathogens, such as Colletotrichum sp. (strawberry) and Fusarium monififorme (Fig. $5 \mathrm{f}$ and Additional file 1: Figure S11).

\section{Discussion}

In our previous study, B. velezensis PG12 was isolated from apple fruit and showed high antifungal activity, which indicates that this bacterial strain has the potential to be used to control fungal pathogens infecting crop plants (Chen et al. 2016). In the present study, we displayed that both $B$. velezensis strains including PG12 and B. siamensis strains contain three secondary metabolism gene clusters related to the production of lipopeptides, such as surfactin, iturin and fengycin. Identification of this large spectrum of antibiotic-biosynthetic genes in the genome of PG12 to some extent explains its 
Table 2 Comparison of COG assignments between the B. amyloliquefaciens group strains

\begin{tabular}{|c|c|c|c|c|c|c|}
\hline \multirow[t]{2}{*}{ Functional categories } & \multicolumn{3}{|c|}{ Core genomes } & \multicolumn{3}{|l|}{$p$-value } \\
\hline & $\mathrm{Bv}$ & $\mathrm{Ba}$ & Bs & $\mathrm{Bv}$ vs $\mathrm{Ba}$ & Bv vs Bs & Ba vs Bs \\
\hline C: Energy production and conversion & 163 & 157 & 163 & 0.9541 & 0.8195 & 0.7734 \\
\hline D: Cell cycle control, cell division, chromosome partitioning & 31 & 30 & 29 & 1 & 0.8974 & 0.8967 \\
\hline E: Amino acid transport and metabolism & 242 & 240 & 232 & 0.8488 & 0.8855 & 0.7361 \\
\hline F: Nucleotide transport and metabolism & 78 & 73 & 78 & 0.8689 & 0.8713 & 0.7413 \\
\hline G: Carbohydrate transport and metabolism & 168 & 157 & 148 & 0.7749 & 0.417 & 0.6372 \\
\hline H: Coenzyme transport and metabolism & 166 & 164 & 163 & 0.9096 & 0.9547 & 0.9545 \\
\hline I: Lipid transport and metabolism & 71 & 72 & 72 & 0.7999 & 0.8654 & 1 \\
\hline $\mathrm{J}$ :Translation, ribosomal structure and biogenesis & 159 & 148 & 164 & 0.7248 & 0.6463 & 0.4141 \\
\hline $\mathrm{K}:$ Transcription & 184 & 177 & 179 & 0.9566 & 1 & 0.9563 \\
\hline L: Replication, recombination and repair & 100 & 98 & 100 & 1 & 0.8855 & 0.9423 \\
\hline M: Cell wall/membrane/envelope biogenesis & 160 & 161 & 158 & 0.863 & 0.9081 & 0.8626 \\
\hline $\mathrm{N}:$ Cell motility & 21 & 20 & 21 & 1 & 1 & 1 \\
\hline O: Posttranslational modification, protein turnover, chaperones & 89 & 86 & 88 & 1 & 0.9392 & 0.9386 \\
\hline P: Inorganic ion transport and metabolism & 145 & 148 & 143 & 0.7185 & 0.9517 & 0.7634 \\
\hline Q: Secondary metabolite biosynthesis, transport and catabolism & 59 & 36 & 37 & 0.02966 & 0.03925 & 1 \\
\hline R: General function prediction only & 300 & 301 & 297 & 0.7301 & 0.8627 & 0.8627 \\
\hline S: Function unknown & 294 & 290 & 289 & 0.8614 & 0.9303 & 0.965 \\
\hline T: Signal transduction mechanism & 80 & 79 & 81 & 0.9359 & 0.8108 & 0.936 \\
\hline U: Intracellular trafficking, secretion and vesicular transport & 26 & 26 & 26 & 1 & 1 & 1 \\
\hline V: Defense mechanism & 46 & 44 & 46 & 1 & 0.9166 & 0.9154 \\
\hline
\end{tabular}

$\mathrm{Bv}$, Ba and Bs represent B. velezensis, B. amyloliquefaciens and B. siamensis, respectively

high competitive ability against other microorganisms. However, it is still not known whether this biocontrol capacity is expressed in the field under different environmental conditions, as biocontrol effectiveness can be critically influenced by the resident microbial communities (Goudjal et al. 2014). Therefore, further field tests on the biocontrol effect of PG12 need to be performed in the future.

The species in the genus of Bacillus are both taxonomically and metabolically diverse, and the taxonomic status of some Bacillus strains constantly brings confusion to researchers (Hamdache et al. 2013). B. subtilis, B. amyloliquefaciens and B. pumilus, used in the agro-food industry, belong to a group of phylogenetically homogeneous species. It is therefore difficult to distinguish these species according to microbiological phenotypic characteristics. Moreover, phylogenetic analysis based on the $16 \mathrm{~S}$ rRNA gene also fails to differentiate these species due to the highly conserved nature of the gene (Rooney et al. 2009). The B. cereus group comprises eleven closely related species, but the phylogenetic and taxonomic relationships within this group are still debated (Liu et al. 2015). With the development of sequencing technology, more and more genomic data of Bacillus strains are released. Construction of phylogenetic trees based on genome sequence will help us to correct errors and improve the taxonomy quality of Bacillus strains. For instance, the biocontrol strains that were ever classified as B. subtilis based on $16 \mathrm{~S}$ rRNA and metabolic profiles were reclassified as B. amyloliquefaciens based on their genome sequence (Magno-Perez-Bryan et al. 2015). On this count, we used 860 single-copy core genes to obtain a robust phylogenetic analysis of the $B$. amyloliquefaciens group strains in this study. The result showed that the tree can be divided into three clusters (Additional file 1: Figure S4). A heat-map analysis based on ANI values also support to divide this group into three subgroups (Fig. 2). With genome sequence data, we can realize accurate identification and taxonomy of Bacillus isolates, and further reveal their distinct features at the molecular level.

The $B$. amyloliquefaciens group harbors a wide range of ecotypes, including soil borne $B$. amyloliquefaciens and plant-associated B. siamensis and B.veleznesis (Reva et al. 2004; Fan et al. 2017a). The most important biocontrol and plant-growth-promoting strains were found in $B$. velezensis, and have been successfully used in agriculture (Borriss 2011). There are significant differences in genome sequences between plant-associated and non-plant-associated strains. Rueckert et al. (2011) performed a genome comparison between FZB42 (a type strain of plant-associated B. velezensis) and DSM7 (a type strain of non-plant-associated B. amyloliquefaciens) 


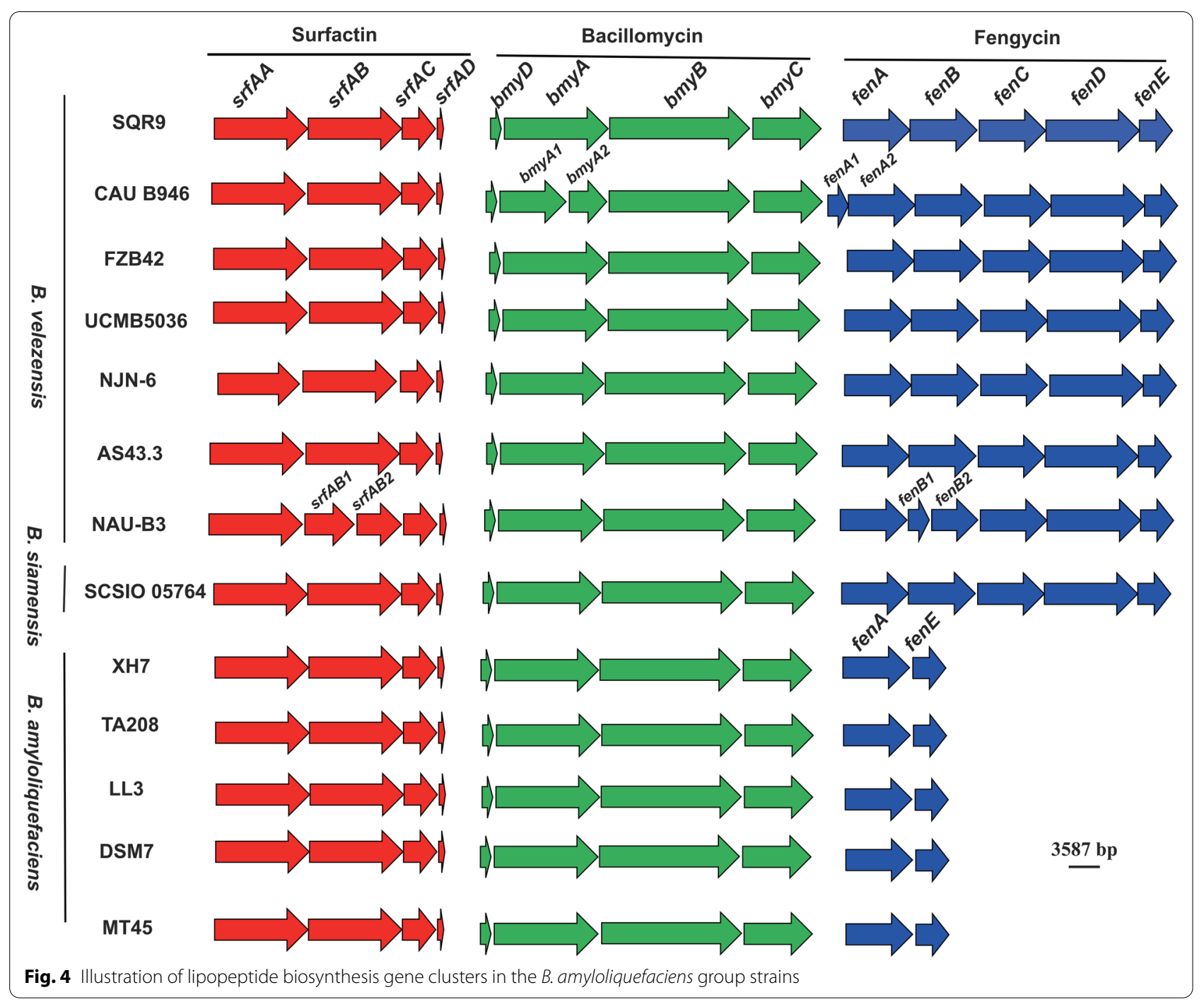

and found significant differences in their genomic sequences (Rueckert et al. 2011). COG function classification and manual curation of the core genomes of the $B$. amyloliquefaciens group strains showed that the genes involved in the biosynthesis of secondary metabolism were more abundant in $B$. velezensis (Table 2). Fan et al. (2017a) also found that the gene cluster involved in macrolactin synthesis is present in B.velezensis, but not in B.siamensis nor in B. amyloliquefaciens. In addition, the number of metabolites produced by $B$. velezensis is higher than those by $B$. siamensis and B. amyloliquefaciens. As a consequence, the strains harboring more antibiotic-synthesizing genes have an increased ability to limit the colonization of crop plants by fungal pathogens through the biosynthesis of antibiotics.

Horizontal gene transfer is recognized as an important factor in driving evolution and differentiation of different organisms. Three of the five genes pertaining to fengycin biosynthesis gene cluster $(f e n B C D)$ were not found in $B$. amyloliquefaciens, whereas were identified in B. siamensis and $B$. velezensis, with the possibility to acquire them from Paenibacillus spp. by HGT. The $\Delta$ fenA mutant of PG12 displayed a significantly decreased antifungal activity, swarming motility and biofilm formation (Fig. 5 and Additional file 1: Figure S11), confirming the key role of this type of lipopeptide in maintaining bacterial antagonistic ability. It also demonstrated that fengycin as an antagonistic compound plays an important role in biocontrol activities (Liu et al. 2021; Xiao et al. 2021). Additionally, fengycin was reported as an elicitor produced by B. velezensis SQR9 and B. subtilis GLB191, and it is able to induce plant immunity by triggering induced systemic resistance in Arabidopsis and grape plants in response to pathogen infection (Wu et al. 2018; Li et al. 2019). Therefore, fengycin-producing strain PG12 is a very promising candidate for the biological control of fungal diseases 
a
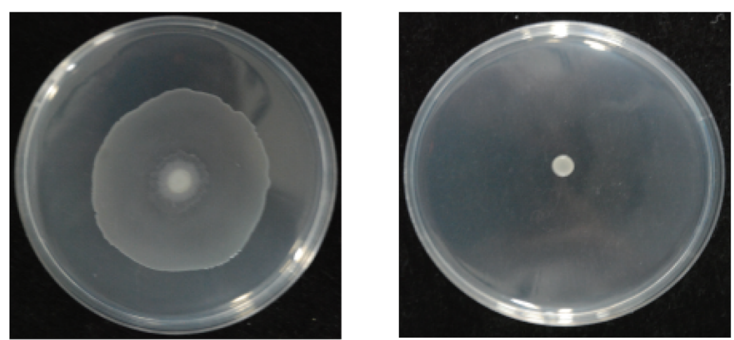

b
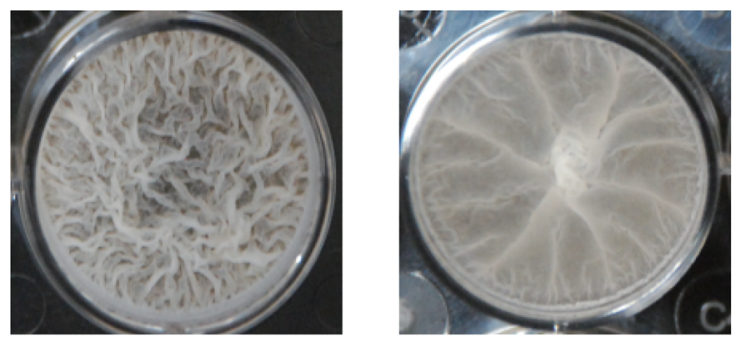

C

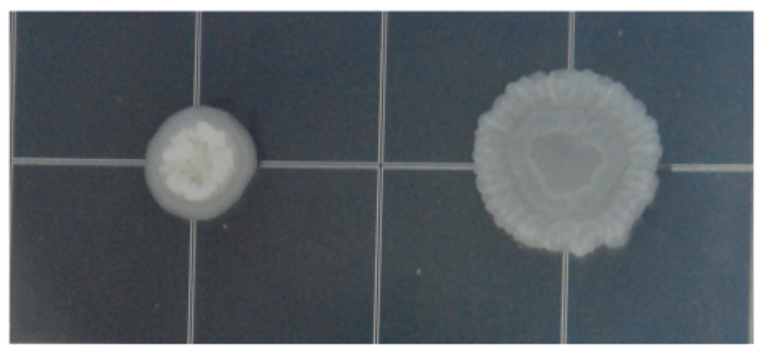

d

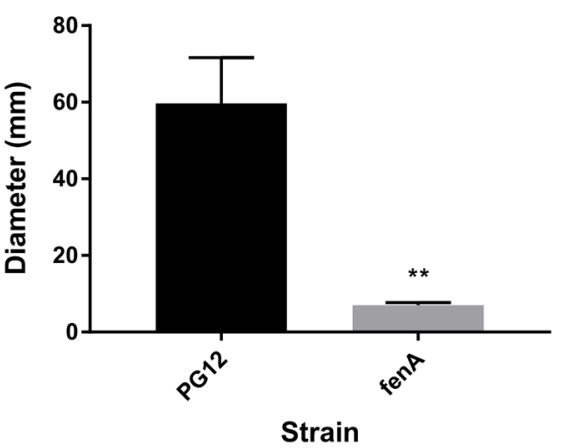

e

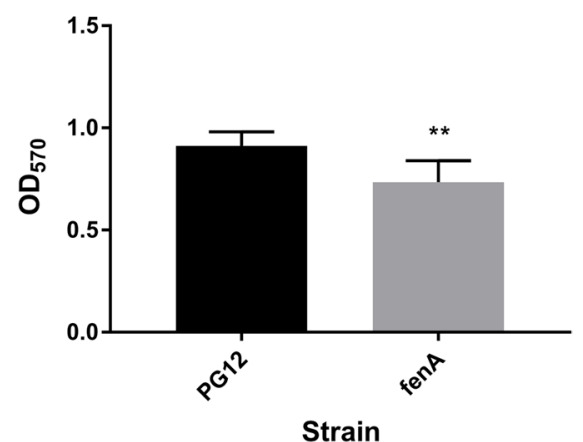

f

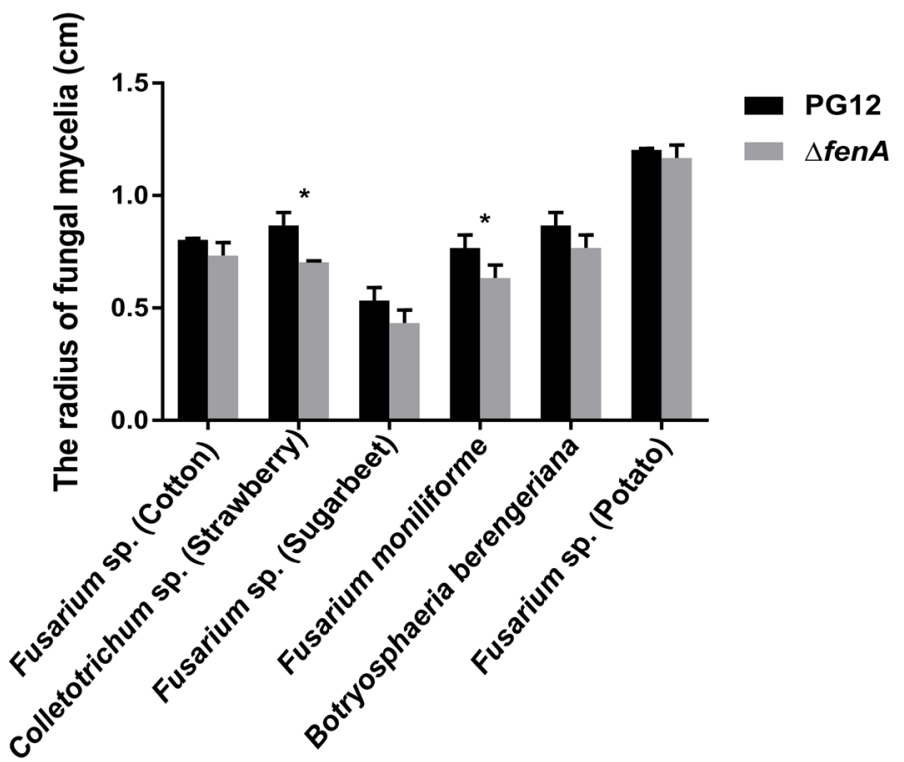

Fig. 5 Comparative analysis of the biocontrol properties of B. velezensis PG12 and $\triangle f e n A$. a The swarming ability of PG12 and $\triangle$ fenA. b Pellicle formation on MSgg liquid media. c Colony morphology on MSgg media. d Quantitative analysis of swarming ability. e Quantitative analysis of biofilm formation. $\mathbf{f}$ Measurement of the inhibition zones of PG12 and $\triangle f e n A$. Data are an average of three replicates \pm the standard deviation of three independent experiments. Bars represent standard deviations. ${ }^{* *}$ and ${ }^{*}$ represent $P<0.01$ and $P<0.05$ (t-test), respectively 
in crop plants. Further studies will also need to characterize the role of the $f e n B C D$ genes and the regulation mechanism of fengycin biosynthesis gene clusters. Altogether, our results promote comparative genomic studies of Bacillus strains, and meanwhile allow to gain insights into the underlying mechanistic basis of their biocontrol activity.

\section{Conclusion}

The biocontrol strain PG12 was identified as B. velezensis based on its genomic sequence. Three lipopeptides gene clusters were identified in the genome of PG12. Furthermore, fengycin was found to contribute to the biocontrol activity of PG12 through maintaining its colonization properties and meanwhile inhibiting pathogen growth. Importantly, genes related to secondary metabolism are more abundant in the core genome of $B$. velezensis. Moreover, B. siamensis and B. velezensis probably acquired the fengycin biosynthesis cluster gene through HGT events during their evolutionary process. Our study offers novel insights into the evolution of the $B$. amyloliquefaciens group strains and also theoretical basis for further application of beneficial strains of this group in agriculture.

\section{Methods}

\section{Strains used in this study}

B. velezensis PG12 was isolated from apple fruit (Chen et al. 2016). Genome data of other Bacillus strains used in this study were downloaded from the National Center for Biotechnology Information (NCBI) database. Fusarium sp., Colletotrichum sp., Botryosphaeria berengeriana and Fusarium moniliforme were provided by the Department of Plant Pathology of the China Agricultural University. All these fungal strains were stored in paraffin at $4^{\circ} \mathrm{C}$ before use.

\section{Genome sequencing, assembly, and annotation}

The draft sequence of the $B$. velezensis PG12 strain was produced using Illumina Hiseq 2500 paired-end (350 bp) sequencing platform by Berry Genomics (Berry Genomics, Beijing). The assembly was performed using SOAPdenovo (v.2.04), resulting in 22 scaffolds for PG12 (Luo et al. 2012; Zeng et al. 2019). Predictions of proteincoding genes were implemented in Prokka (v. 1.11) (Seemann 2014). Functional annotation was carried out using the Basic Local Alignment Search Tool (BLAST) against the Cluster of Orthologous Groups of proteins (COG), NCBI nr protein database, Kyoto Encyclopedia of Genes and Genomes (KEGG) database and InterPro database (Zeng et al., 2018). Ordering of contigs of PG12 was achieved using the program Mauve (Rissman et al. 2009). The genome sequence of B. velezensis CAU B946 was used as a reference sequence. The final annotated chromosome was plotted using CIRCOS software (Krzywinski et al. 2009). The secondary metabolite gene clusters were examined using the antiSMASH v4.0.0rc1 program (Blin et al. 2017).

\section{Phylogenomic analyses}

A ML phylogenetic tree of Bacillus species was constructed based on 624 or 860 single-copy core proteins shared by Bacillus and Paenibacillus polymyxa M1 according to the following methods: (1) multiple alignment of amino acid sequences by mafft (v. 7.310) (Katoh and Standley 2013); (2) conserved blocks from multiple alignment using Gblocks (Castresana 2000); (3) construction of ML tree using RAxML (v. 8.2.10) software and PROTGAMMALGX model with 100 bootstrap replicates (Zeng et al. 2018). The tree was displayed using molecular evolutionary genetic analysis (MEGA). Then, ANI value was calculated using Jspecies software with MUMmer (NUCmer) alignment (Richter and RosselloMora 2009). Single gene alignments were performed with MEGA 5. The neighbor-joining trees were constructed using the same software with 1,000 bootstrap replicates (Kumar et al. 2008).

For genome similarity assessment, genome comparisons were performed by using BRIG program (v. 0.95). The analysis was done by using the blastn option. The genome sequence of $B$. velezensis FZB42 was used as a reference sequence, whereas the genome sequences of strains (CAU B946, PG12 and UCMB5036) were used as query sequences.

\section{Core and pan-genome analyses}

Identification of the core and pan-genomes of the $22 \mathrm{~B}$. amyloliquefaciens group strains were performed with the PGAP pipeline (Zhao et al. 2012). The protein similarity method from PGAP was used to detect a set of core orthologs which were clustered using at least $50 \%$ protein sequence identity to each other and $50 \%$ overlap with the longest sequence with an e-value threshold of $1 \mathrm{e}-5$. The core and strain-specific genomes was extracted from the orthologous table by using a custom Perl script (Zeng et al. 2018). Functional annotation of core genes of $B$. velezensis, B. amyloliquefaciens and B. siamensis was performed using the COG database. The core and pan-genomes, as well as estimated respective sizes and trajectories, were assessed using models and regression algorithms proposed by Tettelin et al. $(2005,2008)$. Both the core and pan-genome were visualized through PanGP (v1.0.1) using default parameters to generate distribution plots of (i) total genes, and (ii) conserved genes found upon progressive sampling of " $n$ " genomes (Zhao et al. 2014). 


\section{Phenotypic characterization of $B$. velezensis PG12 Cultures and conditions}

B. velezensis $\mathrm{PG} 12$ was grown at $37^{\circ} \mathrm{C}$ in Luria-Bertani (LB) broth or on solid LB medium (Chen et al. 2016). For assays of biofilm formation, MSgg medium was used. The recipe for MSgg is as follows: $5 \mathrm{mM}$ potassium phosphate ( $\mathrm{pH} 7.0$ ), $100 \mathrm{mM}$ MOPS (morpholine propane sulfonic acid) ( $\mathrm{pH} 7.0), 2 \mathrm{mM} \mathrm{MgCl}_{2}$, $700 \mathrm{mM} \mathrm{CaCl} 2,50 \mathrm{mM} \mathrm{MnCl}$, $50 \mathrm{mM} \mathrm{FeCl}{ }_{3}, 1 \mathrm{mM}$ $\mathrm{ZnCl}_{2}, 2 \mathrm{mM}$ thiamine, $0.5 \%$ glycerol, $0.5 \%$ glutamic acid, $50 \mathrm{mg} / \mathrm{mL}$ tryptophan, $50 \mathrm{mg} / \mathrm{mL}$ threonine, and $50 \mathrm{mg} / \mathrm{mL}$ phenylalanine (Branda et al. 2001; Fan et al. 2017b). When necessary, the antibiotic kanamycin was added to a final concentration of $50 \mu \mathrm{g} / \mathrm{mL}$.

\section{Construction of fenA deletion mutant}

The fen $A$ deletion mutant was generated from PG12 by homologous recombination. Primers UF/UR and DF/DR were used to amplify the upstream region (1,585 bp) and downstream region (1,500 bp) of fen $A$, respectively. The kanamycin resistance gene was amplified from plasmid pUB110 using primer pair Kan-F/ Kan-R. To construct the knockout vector, the upstream, downstream and resistance gene region were ligated using gene splicing by overlap extension (4,040 bp). Then, the target product was ligated into pMD19-T to generate the knockout plasmid pTFENA, which was subsequently transferred into $B$. velezensis PG12 by electroporation. Kanamycin-sensitive clones were isolated, and the mutants were identified by PCR with primer pair fenA-out-F/fenA-out-R and confirmed by Sanger sequencing (Additional file 2: Table S2, S3).

\section{Analysis of biofilm formation}

Biofilm formation was monitored in MSgg medium. PG12 was grown in $\mathrm{LB}$ medium at $37^{\circ} \mathrm{C}$ overnight. Then, $4 \mu \mathrm{L}$ of cell culture was added to each well of a 12-well microplate, with each well containing $4 \mathrm{~mL}$ of MSgg medium, and incubated statically at $28^{\circ} \mathrm{C}$ for up to $60 \mathrm{~h}$. To quantify biofilm formation, liquid cultures beneath the biofilm were carefully drawn off and the biofilm in each well was washed three times with $1 \mathrm{~mL}$ of sterile saline and fixed with $2 \mathrm{~mL}$ of $99 \%(\mathrm{v} / \mathrm{v})$ methanol for $15 \mathrm{~min}$, followed by air-drying. The dried biofilms were stained with $2 \mathrm{~mL} 0.1 \%$ crystal violet (CV) for $10 \mathrm{~min}$. After excess $\mathrm{CV}$ was removed, the dried biofilms were dissolved with $5 \mathrm{~mL}$ of $33 \%$ (v/v) glacial acetic acid and then were diluted to 10 times for $\mathrm{OD}_{570}$ detection (Weng et al. 2013; Fan et al. 2017b). For colony architecture, $2 \mu \mathrm{L}$ of cell culture was spotted onto the surface of a Petri plate containing MSgg medium and $1.5 \%$ agar, and incubated at $28^{\circ} \mathrm{C}$ for $60 \mathrm{~h}$.

\section{Assays of swarming motility}

The swarming motility of PG12 was tested using standard protocol with minor modification. PG12 cultures in LB liquid medium were prepared with shaking $(200 \mathrm{rpm})$ at $37^{\circ} \mathrm{C}$ to an $\mathrm{OD}_{600}$ of 0.9 , one $\mathrm{mL}$ of cells were collected by centrifugation at $6,000 \mathrm{~g}$ for $5 \mathrm{~min}$, washed with phosphate-buffered saline (PBS), and resuspended in 100 $\mu \mathrm{L}$ PBS. Three $\mu \mathrm{L}$ of cell suspension was spotted on the center of a Petri plate containing LB medium and $0.7 \%$ agar, and the plates were incubated at room temperature for $9 \mathrm{~h}$ to allow cell growth in order to clearly visualize the swarming zone (Chen et al. 2012; Fan et al. 2017b). The plates were air-dried for $2 \mathrm{~h}$ in a laminar flow hood, and then the diameter of the swarming zone was measured.

\section{In vitro antagonism test}

The antagonistic activities of the wild-type and mutant strains against fungal pathogens were assessed as follows. In the plate confrontation assay, the fungi were cultured on potato-dextrose-agar plates (PDA) at $28^{\circ} \mathrm{C}$ for 3-5 days. A 5-mm-diameter mycelial block was then cut from the margin of an actively growing fungal culture and transferred into the center of a fresh PDA plate. After 1 day of incubation, $2 \mu \mathrm{L}$ of overnight bacterial culture grown in LB medium was spotted on the PDA plate $2.5 \mathrm{~cm}$ away from the center where the mycelial block was placed. The antifungal activities of the examined bacterial strains were evaluated by measuring the radial extension of mycelium after $5-7$ days of incubation at $28^{\circ} \mathrm{C}$.

\section{Abbreviations}

ANI: Average nucleotide identity; Blast: Basic local alignment search tool; COG: Cluster of Orthologous Group of proteins; HGT: Horizontal gene transfer; KEGG: Kyoto Encyclopedia of Genes and Genomes; LB: Luria-Bertani; ML: Maximum likelihood; NCBI: National Center for Biotechnology Information; PBS: Phosphate-buffered saline; PDA: Potato dextrose agar.

\section{Supplementary Information}

The online version contains supplementary material available at https://doi. org/10.1186/s42483-021-00103-z.

\footnotetext{
Additional file 1: Figure S1. Genome map of B. velezensis PG12. Each circle has a different genome information, and circles from outside to inside: (1) scale marks (unit: Mb), $(2,3)$ protein-coding genes on the forward and reverse strands, respectively (color-coded by the functional categories), $(4,5)$ rRNA (blue) and tRNA (red) on the forward and reverse strands, respectively, (6) GC content (positive: red; negative: blue), and (7) GC skew (above average: aquamarine; below average: orange). Figure S2. Genome comparisons of FZB42 with other three $B$. velezensis strains. The inner cycle represents the complete genome sequence of FZB42, and the shade of each color shows the similarities between the strains (CAU B946, UCMB5036 or PG12) and FZB42. Figure S3. Venn diagram showing shared orthologous protein clusters in the genomes of PG12 and its closely related B. velezensis strains CAU B946, FZB42 and UCMB5036. Each strain is represented by an oval. The number of orthologous protein-coding genes shared by all strains is in the center. Overlapping regions show the number of coding sequences conserved only within the specified genomes. Numbers in the non-overlapping portions of each oval show
} 
the number of CDS unique to each strain. The total number of proteincoding genes within each genome is listed below the strain name. Figure S4. Phylogenetic tree showing the relationship of the B. amyloliquefaciens group strains. The tree is based on the concatenated alignments of 860 single core genomes. Paenibacillus polymyxa M1 was used as an outgroup. Figure S5. Pan- and core-genome plots of the $B$. amyloliquefaciens group strains. a $B$. velezensis; b $B$. siamensis; $\mathbf{c} B$. amyloliquefaciens. The lines in yellow and purple represent the pan- and core-genomes, respectively. Figure S6. Organization of lipopeptide gene clusters in draft genome sequence of the B. amyloliquefaciens group strains. Different genes are marked with different colors. Figure S7. Comparative analysis of G $+C$ contents between genomes and the fengycin gene clusters (fen $B$, fen $C$ and fenD) in the B. amyloliquefaciens group strains. Figure S8. Neighborjoining phylogenetic tree constructed based on the protein sequences of fenB. Figure S9. Neighbor-joining phylogenetic tree constructed based on the protein sequence of fenC. Figure S10. Neighbor-joining phylogenetic tree constructed based on the protein sequence of fenD. Figure S11. The antifungal activity of PG12 and the $\triangle$ fenA mutant. A mycelium plug of fungal pathogen was inoculated onto the center of a PDA plate, and then $2 \mu \mathrm{L}$ of PG12 and $\triangle$ fenA cultures in $\mathrm{LB}$ medium were added at a distance of $2.5 \mathrm{~cm}$ from the rim of the fungal plug. Clear inhibition zones were observed after incubation for 5-7 days.

Additional file 2: Table S1 Average nucleotide identity pairwise comparisons among the B. amyloliquefaciens group strains. Table S2. Strains and plasmids used in this study. Table S3. Primers used in this study.

\section{Acknowledgements}

We are thankful to Prof. Francis Martin of Beijing Advanced Innovation Center for Tree Breeding by Molecular Design, Beijing Forestry University for help with revising the manuscript.

\section{Authors' contributions}

QZ performed the experiments and wrote the manuscript. QZ, XG and YL analyzed the data. XC, PY and GH prepared the figures and tables. QW and JX helped to design the experiments and revise the manuscript. All authors read and approved the final manuscript.

\section{Funding}

The research was financially supported by the National Natural Science Foundation of China (3162074), the National Key R\&D Program of China (2017YFD0201106; 2019YFD1002003) and the Beijing Municipal Natural Science Foundation (6172018).

\section{Availability of data and materials}

Not applicable.

\section{Declarations}

\section{Ethical approval and consent to participate}

Not applicable.

\section{Consent for publication}

Not applicable.

\section{Competing interests}

The authors declare that they have no competing interests.

\section{Author details}

'Department of Plant Pathology, MOA Key Lab of Pest Monitoring and Green Management, College of Plant Protection, China Agricultural University, Beijing 100193, China. ${ }^{2}$ Beijing Advanced Innovation Center for Tree Breeding by Molecular Design, Beijing Forestry University, Beijing 100083, China. ${ }^{3}$ Institute of Citrus Science, Yichang 443000, China.

Received: 24 June 2021 Accepted: 26 October 2021

Published online: 09 November 2021

\section{References}

Allard-Massicotte R, Tessier L, Lecuyer F, Lakshmanan V, Lucier J, Garneau D, et al. Bacillus subtilis early colonization of Arabidopsis thaliana roots involves multiple chemotaxis receptors. Mbio. 2016;7(6):e01664-16. https://doi.org/10.1128/mBio.01664-16.

Blin K, WolfT, Chevrette MG, Lu X, Schwalen CJ, Kautsar SA, et al. antiSMASH 4.0-improvements in chemistry prediction and gene cluster boundary identification. Nucleic Acids Res. 2017;45:W36-41. https://doi.org/10. 1093/nar/gkx319.

Borriss R. Use of plant-associated Bacillus strains as biofertilizers and biocontrol agents. In: Maheshwari D, editor. Bacteria in Agrobiology: Plant Growth Responses. Heidelberg: Springer. https://doi.org/10.1007/ 978-3-642-20332-9_3.

Branda SS, Gonzalez-Pastor JE, Ben-Yehuda S, Losick R, Kolter R. Fruiting body formation by Bacillus subtilis. Proc Natl Acad Sci USA. 2001;98(20):116216. https://doi.org/10.1073/pnas.191384198.

Castresana J. Selection of conserved blocks from multiple alignments for their use in phylogenetic analysis. Mol Biol Evol. 2000;17(4):540-52. https://doi. org/10.1093/oxfordjournals.molbev.a026334.

Chen Y, Chai Y, Gu J, Losick R. Evidence for cyclic Di-GMP-mediated signaling in Bacillus subtilis. J Bacteriol. 2012;194(18):5080-90. https://doi.org/10.1128/ JB.01092-12.

Chen X, Zhang Y, Fu X, Li Y, Wang Q. Isolation and characterization of Bacillus amyloliquefaciens PG12 for the biological control of apple ring rot. Postharvest Biol Technol. 2016;115:113-21. https://doi.org/10.1016/j.posth arvbio.2015.12.021.

Chun BH, Kim K, Jeong SE, Jeon CO. Genomic and metabolic features of the Bacillus amyloliquefaciens group-B. amyloliquefaciens, B. velezensis, and B. siamensis- revealed by pan-genome analysis. Food Microbiol. 2019;77:146-57. https://doi.org/10.1016/j.fm.2018.09.001.

Cochrane SA, Vederas JC. Lipopeptides from Bacillus and Paenibacillus spp.: a gold mine of antibiotic candidates. Med Res Rev. 2019;36(1):4-31. https:// doi.org/10.1002/med.21321.

Compant S, Duffy B, Nowak J, Clement C, Barka EA. Use of plant growth-promoting bacteria for biocontrol of plant diseases: principles, mechanisms of action, and future prospects. Appl Environ Microbiol. 2005;71(9):49519. https://doi.org/10.1128/AEM.71.9.4951-4959.2005.

Fan B, Blom J, Klenk H, Borriss R. Bacillus amyloliquefaciens, Bacillus velezensis, and Bacillus siamensis form an" operational group B. amyloliquefaciens" within the B. subtilis species complex. Front Microbiol. 2017a;8:22. https:// doi.org/10.3389/fmicb.2017.00022.

Fan H, Zhang Z, Li Y, Zhang X, Duan Y, Wang Q. Biocontrol of bacterial fruit blotch by Bacillus subtilis 9407 via surfactin-mediated antibacterial activity and colonization. Front Microbiol. 2017b;8:1973. https://doi.org/10. 3389/fmicb.2017.01973.

Fan B, Wang C, Song X, Ding X, Wu L, Wu H, et al. Bacillus velezensis FZB42 in 2018: The Gram-positive model strain for plant growth promotion and biocontrol. Front Microbiol. 2019;10:1279. https://doi.org/10.3389/fmicb. 2019.01279.

Feng L, Ma T, Zhang J, Xu F, Shi L. Draft genome sequence of Bacillus subtilis $\mathrm{QH}-1$, a chromium-reducing bacterial strain isolated in Qinghai province. China Genome Announce. 2014;2(2):e00182-e214. https://doi.org/10. 1128/genomeA.00182-14.

Fira D, Dimkic I, Beric T, Lozo J, Stankovic S. Biological control of plant pathogens by Bacillus species. J Biotechnol. 2018;285:44-55. https://doi.org/10. 1016/j.jbiotec.2018.07.044.

Gao T, Ding M, Yang C, Fan H, Chai Y, Li Y. The phosphotransferase system gene ptsH plays an important role in MnSOD production, biofilm formation, swarming motility, and root colonization in Bacillus cereus 905. Res Microbiol. 2019;170(2):86-96. https://doi.org/10.1016/j.resmic.2018.10.002.

Goudjal Y, Toumatia O, Yekkour A, Sabaou N, Mathieu F, Zitouni A. Biocontrol of Rhizoctonia solani damping-off and promotion of tomato plant growth by endophytic actinomycetes isolated from native plants of Algerian Sahara. Microbiol Res. 2014;169(1):59-65. https://doi.org/10.1016/j.micres. 2013.06.014.

Hamdache A, Azarken R, Lamarti A, Aleu J, Collado IG. Comparative genome analysis of Bacillus spp. and its relationship with bioactive nonribosomal peptide production. Phytochem Rev. 2013;12(4):685-716. https://doi.org/ 10.1007/s11101-013-9278-4.

Jayapala N, Mallikarjunaiah NH, Puttaswamy H, Gavirangappa H, Ramachandrappa NS. Rhizobacteria Bacillus spp. induce resistance against 
anthracnose disease in chili (Capsicum annuum L.) through activating host defense response. Egypt J Biol Pest Control. 2019;29:45. https://doi.org/10. 1186/s41938-019-0148-2.

Katoh K, Standley DM. MAFFT: multiple sequence alignment software Version 7: improvements in performance and usability. Mol Biol Evol. 2013;30(4):77280. https://doi.org/10.1093/molbev/mst010.

Kim B, Lee S, Ahn J, Song J, Kim W, Weon H. Complete genome sequence of Bacillus amyloliquefaciens subsp. plantarum CC178, a phyllosphere bacterium antagonistic to plant pathogenic fungi. Genome Announce. 2015;3(1):e1314-68. https://doi.org/10.1128/genomeA.01368-14.

Koumoutsi A, Chen XH, Henne A, Liesegang H, Hitzeroth G, Franke P, et al. Structural and functional characterization of gene clusters directing nonribosomal synthesis of bioactive cyclic lipopeptides in Bacillus amyloliquefaciens strain FZB42. J Bacteriol. 2014;186(4):1084-96. https://doi.org/10.1128/JB. 186.4.1084-1096.2004.

Krzywinski M, Schein J, Birol I, Connors J, Gascoyne R, Horsman D, et al. Circos: an information aesthetic for comparative genomics. Genome Res. 2009;19(9):1639-45. https://doi.org/10.1101/gr.092759.109.

Kumar S, Nei M, Dudley J, Tamura K. MEGA: A biologist-centric software for evolutionary analysis of DNA and protein sequences. Briefings Bioinf. 2008;9(4):299-306. https://doi.org/10.1093/bib/bbn017.

Li Y, Heloir M, Zhang X, Geissler M, Trouvelot S, Jacquens L, et al. Surfactin and fengycin contribute to the protection of a Bacillus subtilis strain against grape downy mildew by both direct effect and defence stimulation. Mol Plant Pathol. 2019;20(8):1037-50. https://doi.org/10.1111/mpp.12809.

Li C, Cheng P, Zheng L, LiY, Chen Y, Wen S, et al. Comparative genomics analysis of two banana Fusarium wilt biocontrol endophytes Bacillus subtilis R31 and TR21 provides insights into their differences on phytobeneficial trait. Genomics. 2021;113(3):900-9. https://doi.org/10.1016/j.ygeno.2021.02.006.

Liu Y, Lai Q, Goeker M, Meier-Kolthoff JP, Wang M, Sun Y, et al. Genomic insights into the taxonomic status of the Bacillus cereus group. Sci Rep. 2015;5:14082. https://doi.org/10.1038/srep14082.

Liu H, Zeng Q, Yalimaimaiti N, Wang W, Zhang R, Yao J. Comprehensive genomic analysis of Bacillus velezensis AL7 reveals its biocontrol potential against Verticillium wilt of cotton. Mol Genet Genomics. 2021. https://doi.org/10. 1007/s00438-021-01816-8.

Luo R, Liu B, Xie Y, Li Z, Huang W, Yuan J, et al. SOAPdenovoz: an empirically improved memory-efficient short-read de novo assembler. GigaScience. 2012. https://doi.org/10.1186/s13742-015-0069-2.

Luo C, Zhou H, Zou J, Wang X, Zhang R, Xiang Y, et al. Bacillomycin L and surfactin contribute synergistically to the phenotypic features of Bacillus subtilis 916 and the biocontrol of rice sheath blight induced by Rhizoctonia solani. Appl Microbiol Biot. 2014;99(4):1897-910. https://doi.org/10.1007/ s00253-014-6195-4.

Magno-Perez-Bryan MC, Martinez-Garcia PM, Hierrezuelo J, Rodriguez-Palenzuela P, Arrebola E, Ramos C, et al. Comparative genomics within the Bacillus genus reveal the singularities of two robust Bacillus amyloliquefaciens biocontrol strains. Mol Plant-Microbe Interact. 2015;28(10):1102-16. https:// doi.org/10.1094/MPMI-02-15-0023-R.

Paterson J, Jahanshah G, Li Y, Wang Q, Mehnaz S, Gross H. The contribution of genome mining strategies to the understanding of active principles of PGPR strains. FEMS Microbiol Ecol. 2017;93:fiw249. https://doi.org/10.1093/ femsec/fiw249.

Prashar P, Kapoor N, Sachdeva S. Biocontrol of plant pathogens using plant growth promoting bacteria. In: Lichtfouse editor. Sustainable agriculture Reviews. Sustainable Agricultural Reviews. Dordrecht: Springer. https://doi. org/10.1007/978-94-007-5961-9_10.

Qiao J, Wu H, Huo R, Gao X, Borriss R. Stimulation of plant growth and biocontrol by Bacillus amyloliquefaciens subsp. plantarum FZB42 engineered for improved action. Chem Biol Technol Agric. 2014;1:12. https://doi.org/10. 1186/s40538-014-0012-2.

Rahman MM. Insecticide substitutes for DDT to control mosquitoes may be causes of several diseases. Environ Sci Pollut Res. 2013;20(4):2064-9. https:// doi.org/10.1007/s11356-012-1145-0.

Reva ON, Dixelius C, Meijer J, Priest FG. Taxonomic characterization and plant colonizing abilities of some bacteria related to Bacillus amyloliquefaciens and Bacillus subtilis. FEMS Microbiol Ecol. 2004;48(2):249-59. https://doi.org/10. 1016/j.femsec.2004.02.003

Richter M, Rossello-Mora R. Shifting the genomic gold standard for the prokaryotic species definition. Proc Natl Acad Sci USA. 2009;106(45):19126-31. https://doi.org/10.1073/pnas.0906412106.
Rissman Al, Mau B, Biehl BS, Darling AE, Glasner JD, Perna NT. Reordering contigs of draft genomes using the Mauve aligner. Bioinformatics. 2009;25(16):2071-3. https://doi.org/10.1093/bioinformatics/btp356.

Rooney AP, Price NPJ, Ehrhardt C, Swezey JL, Bannan JD. Phylogeny and molecular taxonomy of the Bacillus subtilis species complex and description of Bacillus subtilis subsp inaquosorum subsp nov. Int I Syst Evol Microbol. 2009;59(10):2429-36. https://doi.org/10.1099/ijs.0.009126-0.

Rueckert C, Blom J, Chen X, Reva O, Borriss R. Genome sequence of B. amyloliquefaciens type strain DSM7T reveals differences to plant-associated $B$. amyloliquefaciens FZB42. J Biotechnol. 2011;155(1):78-85. https://doi.org/10. 1016/j.jbiotec.2011.01.006.

Schäfer T, Adams T. The importance of microbiology in sustainable agriculture. In: Lugtenberg B. editor. Principles of plant-microbe interactions. Switzerland: Springer. https://doi.org/10.1007/978-3-319-08575-3_2.

Seemann T. Prokka: rapid prokaryotic genome annotation. Bioinformatics. 2014;30(14):2068-9. https://doi.org/10.1093/bioinformatics/btu153.

Siefert JL, Larios-Sanz M, Nakamura LK, Slepecky RA, Paul JH, Moore E, et al. Phylogeny of marine Bacillus isolates from the Gulf of Mexico. Curr Microbiol. 2000;41 (2):84-8. https://doi.org/10.1007/s002840010098.

Tettelin H, Masignani V, Cieslewicz MJ, Donati C, Medini D, Ward NL, et al. Genome analysis of multiple pathogenic isolates of Streptococcus agalactiae: Implications for the microbial "pan-genome." Proc Natl Acad Sci USA. 2005;102(39):13950-5. https://doi.org/10.1073/pnas.0506758102.

Tettelin H, Riley D, Cattuto C, Medini D. Comparative genomics: the bacterial pangenome. Curr Opin Microbiol. 2008;1 1(5):472-7. https://doi.org/10.1016/j. mib.2008.09.006.

Weng J, Wang Y, Li J, Shen Q, Zhang R. Enhanced root colonization and biocontrol activity of Bacillus amyloliquefaciens SQR9 by abrB gene disruption. Appl Microbiol Biot. 2013;97(19):8823-30. https://doi.org/10.1007/ s00253-012-4572-4.

Wu L, Wu H, Qiao J, Gao X, Borriss R. Novel routes for improving biocontrol activity of Bacillus based bioinoculants. Front Microbiol. 2015;6:1395. https://doi.org/ 10.3389/fmicb.2015.01395.

Wu G, Liu Y, Xu Y, Zhang G, Shen Q, Zhang R. Exploring elicitors of the beneficial rhizobacterium Bacillus amyloliquefaciens SQR9 to induce plant systemic resistance and their interactions with plant signaling pathways. Mol Plant-Microbe Interact. 2018;31(5):560-7. https://doi.org/10.1094/ MPMI-11-17-0273-R.

Xiao J, Guo X, Qiao X, Zhang X, Chen X, Zhang D. Activity of fengycin and iturin A isolated from Bacillus subtilis Z-14 on Gaeumannomyces graminis var. tritici and soil microbial diversity. Front Microbiol. 2021;12:682. https://doi.org/10. 3389/fmicb.2021.682437.

Xie J, Du Z, Bai L, Tian C, Zhang Y, Xie J, et al. Comparative genomic analysis of $\mathrm{N}$-2-fixing and non-N-2-fixing Paenibacillus spp.: organization, evolution and expression of the nitrogen fixation genes. PLoS Genet. 2014;10(3):e1004231. https://doi.org/10.1371/journal.pgen.1004231.

Zeng Q, Xie J, Li Y, Gao T, Xu C, Wang Q. Comparative genomic and functional analyses of four sequenced Bacillus cereus genomes reveal conservation of genes relevant to plant-growth-promoting traits. Sci Rep. 2018;8:17009. https://doi.org/10.1038/s41598-018-35300-y.

Zeng Q, Xie J, LiY, Chen X, Wang Q. Draft genome sequence of an endophytic biocontrol bacterium, Bacillus velezensis PG12, isolated from apple fruit. Microbiol Resour Announce. 2019;8(41):e00468-e519. https://doi.org/10. 1128/MRA.00468-19.

Zhao Y, Wu J, Yang J, Sun S, Xiao J, Yu J. PGAP: pan-genomes analysis pipeline. Bioinformatics. 2012;28(3):416-8. https://doi.org/10.1093/bioinformatics/ btr655.

Zhao Y, Jia X, Yang J, Ling Y, Zhang Z, Yu J, et al. PanGP: a tool for quickly analyzing bacterial pan-genome profile. Bioinformatics. 2014;30(9):1297-9. https://doi. org/10.1093/bioinformatics/btu017.

Zhao H, Shao D, Jiang C, Shi J, Li Q, Huang Q, et al. Biological activity of lipopeptides from Bacillus. Appl Microbiol Biot. 2017;101:5951-60. https://doi.org/ 10.1007/s00253-017-8396-0.

\section{Publisher's Note}

Springer Nature remains neutral with regard to jurisdictional claims in published maps and institutional affiliations. 